\title{
Spaser as Nanoscale Quantum Generator and Ultrafast Amplifier
}

\author{
Mark I. Stockman ${ }^{1,2,3}$ \\ 1 Department of Physics and Astronomy, Georgia State University, Atlanta, Georgia 30303, USA \\ 2 Max Planck Institute for Quantum Optics, Hans-Kopfermann-Strasse 1, 85748 Garching, Germany \\ ${ }^{3}$ Ludwig Maximilian University Munich, Am Coulombwall 1, 85748 Garching, Germany
}

(Dated: October 22, 2018)

\begin{abstract}
Nanoplasmonics has recently experienced explosive development with many novel ideas and dramatic achievements in both fundamentals and applications. The spaser has been predicted and observed experimentally as an active element - generator of coherent local fields. Even greater progress will be achieved if the spaser could function as a ultrafast nanoamplifier - an optical counterpart of the MOSFET (metal-oxide-semiconductor field-effect transistor). A formidable problem with this is that the spaser has the inherent feedback causing quantum generation of nanolocalized surface plasmons and saturation and consequent elimination of the net gain, making it unsuitable for amplification. We have overcome this inherent problem and shown that the spaser can perform functions of an ultrafast nanoamplifier in two modes: transient and bistable. On the basis of quantum density matrix (optical Bloch) equations we have shown that the spaser amplifies with gain $\gtrsim 50$, the switching time $\lesssim 100 \mathrm{fs}$ (potentially, $\sim 10 \mathrm{fs}$ ). This prospective spaser technology will further broaden both fundamental and applied horizons of nanoscience, in particular, enabling ultrafast microprocessors working at $10-100 \mathrm{THz}$ clock speed. Other prospective applications are in ultrasensing, ultradense and ultrafast information storage, and biomedicine. The spasers are based on metals and, in contrast to semiconductors, are highly resistive to ionizing radiation, high temperatures, microwave radiation, and other adverse environments.
\end{abstract}

\section{INTRODUCTION}

Nanoplasmonic phenomena (see, e.g., Ref. 1) unfold on the spatial scale between the skin depth $l_{s} \approx 25 \mathrm{~nm}$ (in noble metals) and the nonlocality radius $l_{n l} \sim v_{F} / \omega \sim 1$ nm, where $v_{F}$ is the electron speed at the Fermi surface, and $\omega$ is optical frequency. Nanoplasmonics is ultrafast: the temporal scale of the nanoplasmonic phenomena is between the coherent time of hundred attoseconds defined by the inverse bandwidth of the plasmonic frequency range (between uv and mid ir for plasmonic metals and doped semiconductors $)^{2,3}$ and the surface plasmon (SP) relaxation time $\gamma_{p}^{-1} \sim 10-100$ fs (for noble metals in the visible to near-ir frequency range) 4 . Not just a promise anymore ${ }^{5}$, nanoplasmonics has delivered a number of important applications: ultrasensing 6 , scanning near-field optical microscopy ${ }^{1,7}$, SP-enhanced photodetectors ${ }^{8}$, thermally assisted magnetic recording 9 , generation of extreme uv ${ }^{10}$, biomedical tests ${ }^{6,11}$, SPassisted thermal cancer treatment ${ }^{12}$, and many others.

To continue its vigorous development, nanoplasmonics needs an active device - near-field generator and amplifier of nanolocalized optical fields, which has until recently been absent. A nanoscale amplifier in microelectronics is the metal-oxide-semiconductor field effect transistor (MOSFET) ${ }^{13,14}$, which has enabled all contemporary digital electronics, including computers and communications and formed the present day technology as we know it. However, the MOSFET is limited by frequency and bandwidth to $\lesssim 100 \mathrm{GHz}$, which is already a limiting factor in further technological development. Another limitation of the MOSFET is its high sensitivity to temperature, electric fields, and ionizing radiation, which limits its use in extreme environmental conditions.
An active element of nanoplasmonics is the SPASER (Surface Plasmon Amplification by Stimulated Emission of Radiation) that was proposed $\underline{4}$ (see also Ref. 15) as a nanoscale quantum generator of nanolocalized coherent and intense optical fields. It has recently been observed experimentally 16 . However, there is a formidable problem to set the spaser as a nanoscale quantum amplifier. A principal difference between a laser (quantum optical oscillator) and a quantum optical amplifier is in that the amplifier does not possess feedback: it does not have mirrors and any parasitic feedback due to light scattering should be carefully minimized to prevent spontaneous generation. In a sharp contrast, in the spaser, as we discuss below in this Introduction, the feedback is always inherently present because the metal plasmonic nanoparticle (spaser's core) supports SP modes whose fields exert periodic perturbation on the gain medium causing the feedback, which principally cannot be removed. This feedback will cause the SP generation and the subsequent saturation of the gain. For any spaser (or any laser, for that matter) in a stationary $(\mathrm{CW})$ regime the net amplification must be zero (which is a condition of the stable CW operation). Therefore, one might assume that the spaser cannot serve as a nanoamplifier.

In this article we have solved this problem suggesting two approaches to setting the spaser as a nanoamplifier: (i) Dynamic or transient approach, which based on the fact that during a femtosecond transient process after the population inversion is created but before the CW regime is established, the spaser possesses a net amplification, and (ii) Bistability approach that is based on the inclusion of a saturable absorber in the gain medium, which prevents spontaneous generation and sets the spaser as a bistable (logical) ultrafast nanoamplifier. This is done 
employing a quantum-kinetic theory that is based on density-matrix equations, which are the adaptation of the optical Bloch equations of the laser theory for the case of the spaser. From these equations we have also obtained new and potentially useful results describing not only the ultrafast dynamics of the spaser but also its CW mode: "spasing curve" (that is a relation between the SP population of the spasing mode and the pumping rate) and also the linewidth of the spaser. These results provide an excellent qualitative explanation of the recent experimental data 16 .

On the basis of the present theory, one may envision femtosecond-cycle nanoplasmonic chips with a high degree of integration where spasers communicate and control each other through their local optical fields or are connected with nanoplasmonic wires. These can perform ultrafast microprocessor functions. The spasers can also be integrated with nano-photodetectors and nanosensors to perform complex functions of intelligent ultrafast detection and sensing. In contrast to semiconductor technology, the spasers are based on metals and, therefore, are highly resistive to ionizing radiation, high temperatures and other adverse environments, with possible applications in space, nuclear industries and defense. These functions of spaser will further widen both the fundamental and applied horizons of nanoplasmonics and, generally, science and technology.

\section{A. Spaser Fundamentals}

We have recently discussed the physical principles of the spasing 15 but will briefly reiterate them here for the sake of completeness and self-containment. The spaser is a nanoplasmonic counterpart of the laser ${ }^{4,15}$. The laser has two principal elements: resonator (or cavity) that supports photonic mode(s) and the gain (or active) medium that is population-inverted and supplies energy to the lasing mode(s). An inherent limitation of the laser is that the size of the laser cavity in the propagation direction is at least half wavelength and practically more than that even for the smallest lasers developed $17,18,19$. In the spaser ${ }^{4}$ this limitation is overcome. The spasing modes are surface plasmons (SPs) whose localization length is on the nanoscale $e^{20}$ and is only limited by the minimum inhomogeneity scale of the plasmonic metal and the nonlocality radius ${ }^{21} l_{n l} \sim 1 \mathrm{~nm}$. So, the spaser is truly nanoscopic, whose minimum total size can be just a few $\mathrm{nm}$.

The resonator of a spaser can be any plasmonic metal nanoparticle whose total size $R$ is much less than the wavelength $\lambda$ and whose metal thickness is between $l_{n l}$ and $l_{s}$, which supports a SP mode with required frequency $\omega_{n}$. This metal nanoparticle should be surrounded by the gain medium that overlaps with the spasing SP eigenmode spatially and whose emission line overlaps with this eigenmode spectrally $\underline{\underline{4}}$. As an example, we consider a model of a nanoshell spaser ${ }^{15,22}$, which is illus-

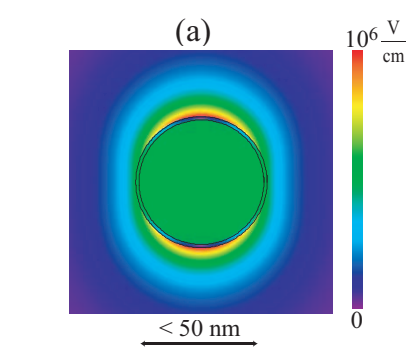

(c)
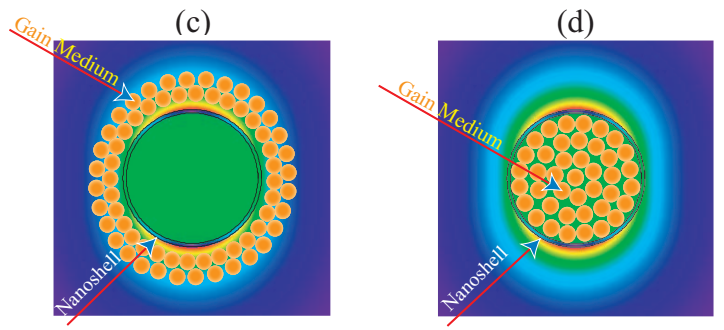

(e)
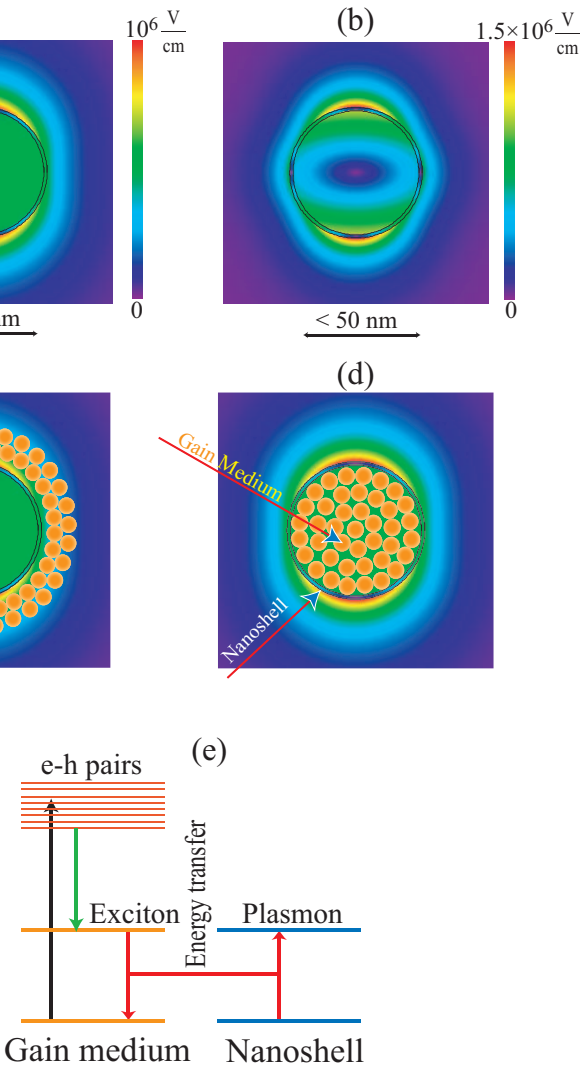

FIG. 1: Schematic of spaser geometry, local fields, and fundamental processes leading to spasing. (a) Nanoshell geometry and the local optical field distribution for one SP in an axially-symmetric dipole mode. The nanoshell has aspect ratio $\eta=0.95$. The local field magnitude is color-coded by the scale bar in the right-hand side of the panel (b) The same as (a) but for a quadrupole mode. (c) Schematic of a nanoshell spaser where the gain medium is outside of the shell, on the background of the dipole-mode field. (d) The same as (c) but for the gain medium inside the shell. (e) Schematic of the spasing process. The gain medium is excited and populationinverted by an external source, as depicted by the black arrow, which produces electron-hole pairs in it. These pairs relax, as shown by the green arrow, to form the excitons. The excitons undergo decay in the ground state emitting SPs in the nanoshell. The plasmonic oscillations of the nanoshell stimulates this emission, supplying the feedback for the spaser action.

trated in Fig. 1, Panel (a) shows a silver nanoshell carrying a single SP (plasmon population number $N_{n}=1$ ) in the dipole eigenmode. It is characterized by a uniform field inside the core and hot spots at the poles outside the shell with the maximum field reaching $\sim 10^{6} \mathrm{~V} / \mathrm{cm}$. Similarly, Fig. 1 (b) shows the quadrupole mode in the same nanoshell. In this case, the mode electric field is non-uniform, exhibiting hot spots of $\sim 1.5 \times 10^{6} \mathrm{~V} / \mathrm{cm}$ of the modal electric field at the poles. These high values of the modal fields is the underlying physical reason for very strong feedback in the spaser. Under our conditions, the electromagnetic retardation within the spaser volume can 
be safely neglected. Also, the radiation of such a spaser is a weak effect: the decay rate of plasmonic eigenmodes is dominated by the internal loss in the metal. Therefore, it is sufficient to consider only quasistatic eigenmodes 20,23 and not their full electrodynamic counterparts ${ }^{24}$, which also is necessary for the ultrafast operation of the spaser as a nanoamplifier.

Note that for high aspect ratios (say, $\eta=0.95$ considered in some of the examples) and relatively small radii $(R=12 \mathrm{~nm})$ the thickness of the metal shell may be rather small (less than $1 \mathrm{~nm}$ ), which may lead to nonlocal effects including Landau damping. In this case, the radius of the spaser can be safely increased by a factor of 2 to 3 (this will not adversely effect the spasing as the spaser is fully scalable, as we show below in Sec. IIB. Also one should keep in mind that the nonlocal effects in thin nanoshells are small due to the special symmetry reasons 25 . We also consider a number of examples with significantly higher aspect ratios where the metal thickness is a few nanometers or larger.

For the sake of numerical illustrations of our theory, we will use the dipole eigenmode [Fig. 1 (a)]. There are two basic ways to place the gain medium: (i) outside the nanoshell, as shown in panel (c), and (ii) in the core, as in panel (d), which was originally proposed in Ref. 22. As we have verified, these two designs lead to comparable characteristics of the spaser. However, the placement of the gain medium inside the core illustrated in Fig. 1 (d) has a significant advantage because the hot spots of the local field are not covered by the gain medium and are sterically available for applications.

Note that any $l$-multipole mode of a spherical particle is, indeed, $2 l+1$-times degenerate. This may make the spasing mode to be polarization unstable, like in lasers without polarizing elements. In reality, the polarization may be pinned and become stable due to deviations from the perfect spherical symmetry, which exist naturally or can be introduced deliberately. More practical shape for a spaser may be a nanorod, which has a mode with the stable polarization along the major axis. However, a nanorod is a more complicated geometry for theoretical treatment, and we will consider it elsewhere.

The level diagram of the spaser gain medium and the plasmonic metal nanoparticle is displayed in Fig. 11 (e) along with a schematic of the relevant energy transitions in the system. The gain medium chromophores may be semiconductor nanocrystals $\$ .26$, dye molecules 27,28 , rare-earth ions 22 , or electron-hole excitations of a bulk semiconductor ${ }^{17,18}$. For certainty, we will use a semiconductor-science language of electrons and holes.

The pump excites electron-hole pairs in the chromophores [Fig. 10(e)], as indicated by the vertical black arrow, which relax to form excitons. The excitons constitute the two-level systems that are the donors of energy for the SP emission into the spasing mode. In vacuum, the excitons would recombine emitting photons. However, in the spaser geometry, the photoemission is strongly quenched due to the resonance energy transfer to the SP modes, as indicated by the red arrows in the panel. The plasmons in the spaser mode create the high local fields that excite the gain medium and stimulate more emission to this mode, which is the feedback mechanism. If this feedback is strong enough and the life time of the spaser SP mode is long enough, then an instability develops leading to the avalanche of the SP emission in the spasing mode and spontaneous symmetry breaking, establishing the phase coherence of the spasing state. Thus the establishment of spasing can be called a nonequilibrium phase transition, as in the physics of lasing.

\section{B. Brief Overview of Latest Progress in Spasers and Nanolasers}

After the original theoretical proposal and prediction of the spaser ${ }^{4}$, there has been an active development in this field, both theoretical and experimental. We comment below only on some representative publications. Among theoretical developments, a nanolens spaser has been proposed ${ }^{29}$, which possesses a nanofocus ("the hottest spot") of the local fields. In Refs. 4, 29, only the necessary condition of spasing has been established on the basis of the perturbation theory.

There have been theories published describing the spaser (or, "nanolaser" as sometimes it is called) phenomenologically, on the basis of classic linear electrodynamics by considering the gain medium as a dielectric with a negative imaginary part of the permittivity 22,30,31.32. Such electrodynamic approaches do not take into account the nature of the spasing as a spontaneous symmetry breaking. This leads to principal differences of their results from the present microscopic quantum-mechanical theory in the region of spasing, as we discuss below in Sec. IIIA in conjunction with Fig. 2. Note that before the spasing threshold, the abovementioned phenomenological theories are, in principle, applicable. There has also been a theoretical publication on a bowtie spaser (nanolaser) with electrical pumping 33 . It is based on balance equations and only the CW spasing generation intensity is described. Yet another theoretical development has been a proposal of the lasing spaser ${ }^{34}$, which is made of a plane array of spasers. The theoretical publications mentioned above in this paragraph deal with the $\mathrm{CW}$ regime. In contrast, in this article we are most interested in the ultrafast behavior of the spaser.

There have also been a theoretical proposal of a spaser ("nanolaser") consisting of a metal nanoparticle coupled to a single chromophore ${ }^{35}$. In this paper, a dipole-dipole interaction is illegitimately used at very small distances $r$ where it has a singularity (diverging for $r \rightarrow 0$ ), leading to a dramatically overestimated coupling with the SP mode. As a result, a completely unphysical prediction of CW spasing due to single chromophore has been obtained ${ }^{35}$. In contrast, our theory is based on the full 
(exact) field of the spasing SP mode. As our results of Sec. III below show, hundreds of chromophores per metal nanoparticle are realistically requited for the spasing even under the most favorable conditions.

There has been a vigorous experimental quest toward the spaser. Stimulated emission of SPPs has been observed in a proof-of-principle experiment using pumped dye molecules as an active (gain) medium ${ }^{27}$. There have also been later experiments that demonstrated strong stimulated emission compensating a significant part of the SPP loss $28,36,37,38$. As a step toward the lasing spaser, the first experimental demonstration has been reported of compensating Joule losses in a metallic photonic metamaterial using optically pumped $\mathrm{PbS}$ semiconductor quantum $\operatorname{dots}^{26}$. In another development, a tunable free-electron light nanosource ("light well") has been demonstrated ${ }^{39}$, which is based on spontaneous emission in a periodically layered metal-dielectric structure. There have also been experimental investigations reporting the stimulated emission effects of SPs in plasmonic metal nanoparticles surrounded by gain media with dye molecules 40,41 . An electrically-pumped nanolaser with semiconductor gain medium has been demonstrated ${ }^{18}$ where the lasing modes are SPPs. A nanolaser with optically-pumped semiconductor gain medium and a hybrid semiconductor-silver SPP waveguide has been demonstrated with an extremely tight transverse mode confinement $\underline{19}$.

Finally, an observation has been reported of a true spaser by M. Noginov et al $\stackrel{16}{\underline{16}}$. This spaser is a chemically synthesized gold nanosphere of radius $7 \mathrm{~nm}$ surrounded by a dielectric shell of a $21 \mathrm{~nm}$ outer radius containing immobilized dye molecules, which is sometimes referred to as "Cornell nanodot". Under nanosecond optical pumping in the absorption band of the dye, this spaser develops a relatively narrow-spectrum and intense visible emission that exhibits a pronounced threshold in pumping intensity. The observed characteristics of this spaser are in an excellent qualitative agreement and can be fully understood on the basis of the corresponding theoretical results obtained below in Sec. IIIA

This article is organized as the following. In Sec. IIA for the case of the spaser, we formulate quantum density matrix equations (also called in the literature the optical Bloch equations). In Sec. IIB we consider these equations for a stationary $(\mathrm{CW})$ case where we find the conditions of spasing, the population of the SPs in the spasing mode, and the spasing line shape. In Sec. III A we illustrate the spasing curve and spectral composition of the spaser generation. In Sec. IIIA we describe the CW spaser as a bistable nanodevice. The major results of this articles regarding the ultrafast kinetics of the spaser as a generator and quantum nanoamplifier are presented in Sec. IV] We briefly discuss the obtained results and conclude in Sec. $\nabla$.

\section{EQUATIONS OF SPASER}

\section{A. Quantum Density Matrix (Optical Bloch) Equations for Spaser}

The SP eigenmodes $\varphi_{n}(\mathbf{r})$ are described by a wave equation (with homogeneous boundary conditions) $)^{4,20}$

$$
\nabla \Theta(\mathbf{r}) \nabla \varphi_{n}(\mathbf{r})=s_{n} \nabla^{2} \varphi_{n}(\mathbf{r})
$$

where $n$ is the mode number, $s_{n}$ is corresponding eigenvalue, and $\Theta(\mathbf{r})$ is the characteristic function equal to 1 for $\mathbf{r}$ in the metal component and 0 in the dielectric. Note that the eigenvalues $s_{n}$ are all real and contained in the range $1 \geq s_{n} \geq 0$. The eigenmodes are normalized by an integral over the volume $V$ of the system, $\int_{V}\left|\nabla \varphi_{n}(\mathbf{r})\right|^{2} d^{3} r=1$. The physical frequency $\omega_{n}$ of the SPs is defined by an equation $\operatorname{Re}\left[s\left(\omega_{n}\right)\right]=s_{n}$, where $s(\omega)=\varepsilon_{d} /\left[\varepsilon_{d}-\varepsilon_{m}(\omega)\right]$ is Bergman's spectral parameter, $\varepsilon_{d}$ is the permittivity of the ambient dielectric, and $\varepsilon_{m}(\omega)$ is the metal permittivity.

The electric field operator ${ }^{45}$ of the quantized SPs is ${ }^{4}$

$\mathbf{E}(\mathbf{r})=-\sum_{n} A_{n} \nabla \varphi_{n}(\mathbf{r})\left(\hat{a}_{n}+\hat{a}_{n}^{\dagger}\right), \quad A_{n}=\left(\frac{4 \pi \hbar s_{n}}{\varepsilon_{d} s_{n}^{\prime}}\right)^{1 / 2}$,

where $\hat{a}_{n}^{\dagger}$ and $\hat{a}_{n}$ are the SP creation and annihilation operators, and $s_{n}^{\prime}=\operatorname{Re}\left[d s\left(\omega_{n}\right) / d \omega_{n}\right]$.

The spaser Hamiltonian has the form

$$
H=H_{g}+\hbar \sum_{n} \omega_{n} \hat{a}_{n}^{\dagger} \hat{a}_{n}-\sum_{p} \mathbf{E}\left(\mathbf{r}_{p}\right) \mathbf{d}^{(p)},
$$

where $H_{g}$ is the Hamiltonian of the gain medium, $p$ is an index (label) of a gain medium chromophore, $\mathbf{r}_{p}$ is its coordinate vector, and $\mathbf{d}^{(p)}$ is its dipole moment operator. In this paper, we will treat the active medium quantum mechanically but the SPs quasiclassically, considering $\hat{a}_{n}$ as a classical quantity (c-number) $a_{n}$ with time dependence as $a_{n}=a_{0 n} \exp (-i \omega t)$, where $a_{0 n}$ is a slowly-varying amplitude. The number of coherent SPs per spasing mode is then given by $N_{p}=\left|a_{0 n}\right|^{2}$. This approximation neglects the quantum fluctuations of the SP amplitudes. However, when necessary, we will take into account these quantum fluctuations, in particular, to describe the spectrum of the spaser.

Introducing $\rho^{(p)}$ as the density matrix of a $p$ th chromophore, we can find its equation of motion in a conventional way by commutating it with the Hamiltonian (3) as $i \hbar \dot{\rho}^{(p)}=\left[\rho^{(p)}, H\right]$, where the dot denotes temporal derivative. We will use the standard rotating wave approximation (RWA), which only takes into account the resonant interaction between the optical field and chromophores. We denote $|1\rangle$ and $|2\rangle$ as the ground and excited states of a chromophore, with the transition $|2\rangle \rightleftharpoons|1\rangle$ resonant to the spasing plasmon mode $n$. In this approximation, the time dependence of the nondiagonal elements of the density matrix is $\left(\rho^{(p)}\right)_{12}=\bar{\rho}_{12}^{(p)} \exp (i \omega t)$, 
and $\left(\rho^{(p)}\right)_{21}=\bar{\rho}_{12}^{(p) *} \exp (-i \omega t)$, where $\bar{\rho}_{12}^{(p)}$ is a timeindependent amplitude defining the coherence (polarization) for the $|2\rangle \rightleftharpoons|1\rangle$ spasing transition in a $p$ th chromophore of the gain medium.

Introducing a rate constant $\Gamma_{12}$ to describe the polarization relaxation and a difference $n_{21}^{(p)}=\rho_{22}^{(p)}-\rho_{11}^{(p)}$ as the population inversion on this spasing transition, we derive an equation of motion for the non-diagonal element of the density matrix as

$$
\dot{\bar{\rho}}_{12}^{(p)}=-\left[i\left(\omega-\omega_{12}\right)+\Gamma_{12}\right] \bar{\rho}_{12}^{(p)}+i n_{21}^{(p)} \Omega_{12}^{(a) *},
$$

where $\Omega_{12}^{(p)}=-A_{n} \mathbf{d}_{12}^{(p)} \nabla \varphi_{n}\left(\mathbf{r}_{p}\right) a_{0 n} / \hbar$ is the Rabi frequency for the spasing transition in a $p$ th chromophore, and $\mathbf{d}_{12}^{(p)}$ is the corresponding transitional dipole element. Note that always $\mathbf{d}_{12}^{(p)}$ is either real or can be made real by a proper choice of the quantum state phases, making the Rabi frequency $\Omega_{12}^{(p)}$ also a real quantity.

An equation of motion for $n_{21}^{p}$ can be found in a standard way by commutating it with $H$. To provide conditions for the population inversion $\left(n_{21}^{p}>0\right)$, we imply existence of a third level. For simplicity, we assume that it very rapidly decays into the excited state $|2\rangle$ of the chromophore, so its own populations is negligible. It is pumped by an external source from the ground state (optically or electrically) with some rate that we will denote $g$. In this way, we obtain the following equation of motion:

$$
\dot{\bar{n}}_{21}^{(p)}=-4 \operatorname{Im}\left[\bar{\rho}_{12}^{(p)} \Omega_{21}^{(p)}\right]-\gamma_{2}\left(1+n_{21}^{(p)}\right)+g\left(1-n_{21}^{(p)}\right),
$$

where $\gamma_{2}$ is the decay rate $|2\rangle \rightarrow|1\rangle$.

The stimulated emission of the SPs is described as their excitation by the coherent polarization of the gain medium. The corresponding equation of motion can be obtained using Hamiltonian (3) and adding the SP relaxation with a rate of $\gamma_{n}$ as

$$
\dot{a}_{0 n}=\left[i\left(\omega-\omega_{n}\right)-\gamma_{n}\right] a_{0 n}+i \sum_{p} \rho_{12}^{(p) *} \Omega^{(p)_{12}} .
$$

Another relevant process is spontaneous emission of SPs by a chromophore into a spasing SP mode. The corresponding rate $\gamma_{2}^{(p)}$ for a chromophore at a point $\mathbf{r}_{p}$ can be found in a standard way using the quantized field (2) as

$$
\gamma_{2}^{(p)}=2 \frac{A_{n}^{2}}{\hbar \gamma_{n}}\left|\mathbf{d}_{12} \nabla \varphi_{n}\left(\mathbf{r}_{p}\right)\right|^{2} \frac{\left(\Gamma_{12}+\gamma_{n}\right)^{2}}{\left(\omega_{12}-\omega_{n}\right)^{2}+\left(\Gamma_{12}+\gamma_{n}\right)^{2}} .
$$

As in Schawlow-Towns theory of laser-line width, this spontaneous emission of SPs leads to the diffusion of the phase of the spasing state. This defines width $\gamma_{s}$ of the spasing line as

$$
\gamma_{s}=\frac{\sum_{p}\left(1+n_{21}^{(p)}\right) \gamma_{2}^{(p)}}{2\left(2 N_{p}+1\right)} .
$$

This width is small for a case of developed spasing when $N_{p} \gg 1$. However, for $N_{p} \sim 1$, the predicted width may be too high because the spectral diffusion theory assumes that $\gamma_{s} \lesssim \gamma_{n}$. To take into account this limitation in a simple way, we will interpolate to find the resulting spectral width $\Gamma_{s}$ of the spasing line as $\Gamma_{s}=\left(\gamma_{n}^{-2}+\gamma_{s}^{-2}\right)^{-1 / 2}$.

We will also examine the spaser as a bistable (logical) amplifier. One of the ways to set the spaser in such a mode is to add a saturable absorber. This is described by the same Eqs. (4)-(6) where the chromophores belonging to the absorber are not pumped by the external source directly, i.e., for them in Eq. (5) one has to set $g=0$.

Numerical examples are given for a silver nanoshell where the core and the external dielectric have the same permittivity of $\varepsilon_{d}=2$; the permittivity of silver is adopted from Ref. 42. The following realistic parameters of the gain medium are used (unless indicated otherwise): $d_{12}=1.5 \times 10^{-17} \mathrm{esu}, \hbar \Gamma_{12}=10 \mathrm{meV}, \gamma_{2}=4 \times 10^{12} \mathrm{~s}^{-1}$ (this value takes into account the spontaneous decay into SPs), and density of the gain medium chromophores is $\rho=2.4 \times 10^{20} \mathrm{~cm}^{-3}$, which is realistic for dye molecules but may be somewhat high for semiconductor quantum dots that were proposed as the chromophores $\underline{\underline{4}}$ and used in experiments 26 . We will assume a dipole SP mode and chromophores situated in the core of the nanoshell as shown in Fig. 1(d). This configuration are of advantage both functionally (because the region of the high local fields outside the shell is accessible for various applications) and computationally (the uniformity of the modal fields makes the summation of the chromophores trivial, thus greatly facilitating numerical procedures).

\section{B. Equations for Stationary (CW) Regime}

Physically, the spaser action is a result of spontaneous symmetry breaking when the phase of the coherent SP field is established from the spontaneous noise. Mathematically, the spaser is described by homogeneous differential Eqs. (4)-(6). These equations become homogeneous algebraic equations for the stationary [or continuous wave $(\mathrm{CW})]$ case. They always have a trivial, zero solution. However, when their determinant vanishes, they also possess a nontrivial solution describing spasing, whose condition is

$$
\begin{aligned}
& \left(\omega_{s}-\omega_{n}+i \gamma_{n}\right)^{-1} \times \\
& \left(\omega_{s}-\omega_{21}+i \Gamma_{12}\right)^{-1} \sum_{p}\left|\tilde{\Omega}_{12}^{(p)}\right|^{2} n_{21}^{(p)}=-1,
\end{aligned}
$$

where $\tilde{\Omega}_{12}^{(p)}=-A_{n} \mathbf{d}_{12}^{(p)} \nabla \varphi_{n}\left(\mathbf{r}_{p}\right) / \hbar$ is the single-plasmon Rabi frequency. The population inversion of a $p$ th chromophore $n_{21}^{(p)}$ is explicitly expressed as

$$
\begin{aligned}
& n_{21}^{(p)}=\left(g-\gamma_{2}\right) \times \\
& \left\{g+\gamma_{2}+4 N_{n}\left|\tilde{\Omega}_{12}^{(p)}\right|^{2} /\left[\left(\omega_{s}-\omega_{21}\right)^{2}+\Gamma_{12}^{2}\right]\right\}^{-1} .
\end{aligned}
$$


From the imaginary part of Eq. (10) we immediately find the spasing frequency

$$
\omega_{s}=\left(\gamma_{n} \omega_{21}+\Gamma_{12} \omega_{n}\right) /\left(\gamma_{n}+\Gamma_{12}\right),
$$

which generally does not coincide with either the gain transition frequency $\omega_{21}$ or the SP frequency $\omega_{n}$, but is between them (this is a frequency walk-off phenomenon similar to that of laser physics). Substituting Eq. (11) back to Eqs. (10)-(11), we obtain a system of equations

$$
\begin{aligned}
& \frac{\left(\gamma_{n}+\Gamma_{12}\right)^{2}}{\gamma_{n} \Gamma_{12}\left[\left(\omega_{21}-\omega_{n}\right)^{2}+\left(\Gamma_{12}+\gamma_{n}\right)^{2}\right]} \times \\
& \sum_{p}\left|\tilde{\Omega}_{12}^{(p)}\right|^{2} n_{21}^{(p)}=1, \\
& n_{21}^{(p)}=\left(g-\gamma_{2}\right) \times \\
& {\left[g+\gamma_{2}+\frac{4 N_{n}\left|\tilde{\Omega}_{12}^{(p)}\right|^{2}\left(\Gamma_{12}+\gamma_{n}\right)}{\left(\omega_{12}-\omega_{n}\right)^{2}+\left(\Gamma_{12}+\gamma_{n}\right)^{2}}\right]^{-1} .}
\end{aligned}
$$

This system defines the stationary $(\mathrm{CW})$ number of SPs per spasing mode $N_{n}$.

Since $n_{21}^{(p)} \leq 1$, from Eqs. (12), (13) we immediately obtain a necessary condition of the existence of spasing,

$$
\frac{\left(\gamma_{n}+\Gamma_{12}\right)^{2}}{\gamma_{n} \Gamma_{12}\left[\left(\omega_{21}-\omega_{n}\right)^{2}+\left(\Gamma_{12}+\gamma_{n}\right)^{2}\right]} \sum_{p}\left|\tilde{\Omega}_{12}^{(p)}\right|^{2} \geq 1 .
$$

This expression is fully consistent with Ref. 4. The following order of magnitude estimate of this spasing condition has a transparent physical meaning and is of heuristic value,

$$
\frac{d_{12}^{2} Q N_{c}}{\hbar \Gamma_{12} V_{n}} \gtrsim 1
$$

where $Q=\omega / \gamma_{n}$ is the quality factor of SPs, $V_{n}$ is the volume of the spasing SP mode, and $N_{c}$ is the of number of the gain medium chromophores within this volume. Deriving this estimate, we have neglected the detuning, i.e., set $\omega_{21}-\omega_{n}=0$. We also used the definitions of $A_{n}$ of Eq. (2) and $\tilde{\Omega}_{12}^{(p)}$ given after Eq. (10), and the estimate $\left|\nabla \varphi_{n}(\mathbf{r})\right|^{2} \sim 1 / V$ following from the normalization of the SP eigenmodes $\int\left|\nabla \varphi_{n}(\mathbf{r})\right|^{2} d^{3} r=1$ of Ref. 20. The result of Eq. (15) is, indeed, in agreement with Ref. 4 where it was obtained in slightly different notations.

It follows from Eq. (15) that for the existence of spasing it is beneficial to have a high quality factor $Q$, a high density of the chromophores, and a large transition dipole (oscillator strength) of the chromophore transition. The small modal volume $V_{n}$ (at a given number of the chromophores $N_{c}$ ) is beneficial for this spasing condition: physically, it implies strong feedback in the spaser. Note that for the given density of the chromophores $\rho_{c}=N_{c} / V_{n}$, this spasing condition does not explicitly depend on the spaser size, which opens up a possibility of spasers of a very small size limited from the bottom by only the nonlocality radius $l_{n l} \sim 1 \mathrm{~nm}$. Another important property of Eq. (15) is that it implies the quantum-mechanical nature of spasing and spaser amplification: this condition essentially contains the Planck constant $\hbar$ and, thus, does not have a classical counterpart. Note that in contrast to lasers, the spaser theory and Eq. (15) in particular do not contain speed of light, i.e., they are quasistatic.

\section{SPASER IN MODE OF CONTINUOUS WAVE NANOSCALE QUANTUM GENERATOR}

\section{A. Kinetics of CW Spaser}

The "spasing curve", i.e., the dependence of the coherent SP population $N_{n}$ on the excitation rate $g$, obtained by solving Eqs. (12), (13), is shown in Fig. 2 (a) for four types of the silver nanoshells with the frequencies of the spasing dipole modes as indicated, which are in the range from near ir $\left(\hbar \omega_{s}=1.2 \mathrm{eV}\right)$ to mid visible $\left(\hbar \omega_{s}=2.2 \mathrm{eV}\right)$. In all cases, there is a pronounced threshold of the spasing at an excitation rate $g_{t h} \sim 10^{12} \mathrm{~s}^{-1}$. Soon after the threshold, the dependence $N_{n}(g)$ becomes linear, which means that every quantum of excitation added to the active medium with a high probability is stimulated to be emitted as a SP, adding to the coherent SP population.

While this is similar to conventional lasers, there is a dramatic difference for the spaser. In the lasers a similar relative rate of the stimulated emission is achieved at a photon population of $\sim 10^{18}-10^{20}$, while in the spaser the SP population is $N_{n} \lesssim 100$. This is due to the much stronger feedback in spasers because of the much smaller modal volume $V_{n}$ - see discussion of Eq. (15). The shape of the spasing curves of Fig. 2 (a) (the well-pronounced threshold with the linear dependence almost immediately above the threshold) is in an excellent qualitative agreement with the experiment 16 . Note that the recent SPP nanolaser with a tight transverse confinement of the lasing modes does not exhibit a pronounced threshold $\frac{19}{9}$, in contrast to the spaser.

The population inversion number $n_{21}$ as a function of the excitation rate $g$ is displayed in Fig. 2 (b) for the same set of frequencies (and with the same color coding) as in panel (a). Before the spasing threshold, $n_{21}$ increases with $g$ to become positive with the onset of the population inversion just before the spasing threshold. For higher $g$, after the spasing threshold is exceeded, the inversion $n_{21}$ becomes constant (the inversion pinning). The pinned levels of the inversion are very low, $n_{21} \sim 0.01$, which again is due to the very strong feedback in the spaser.

The spectral width $\Gamma_{s}$ of the spaser generation is due to the phase diffusion of the quantum SP state caused by the noise of the spontaneous emission of the SPs into the spasing mode, as described by Eq. (8). This width 

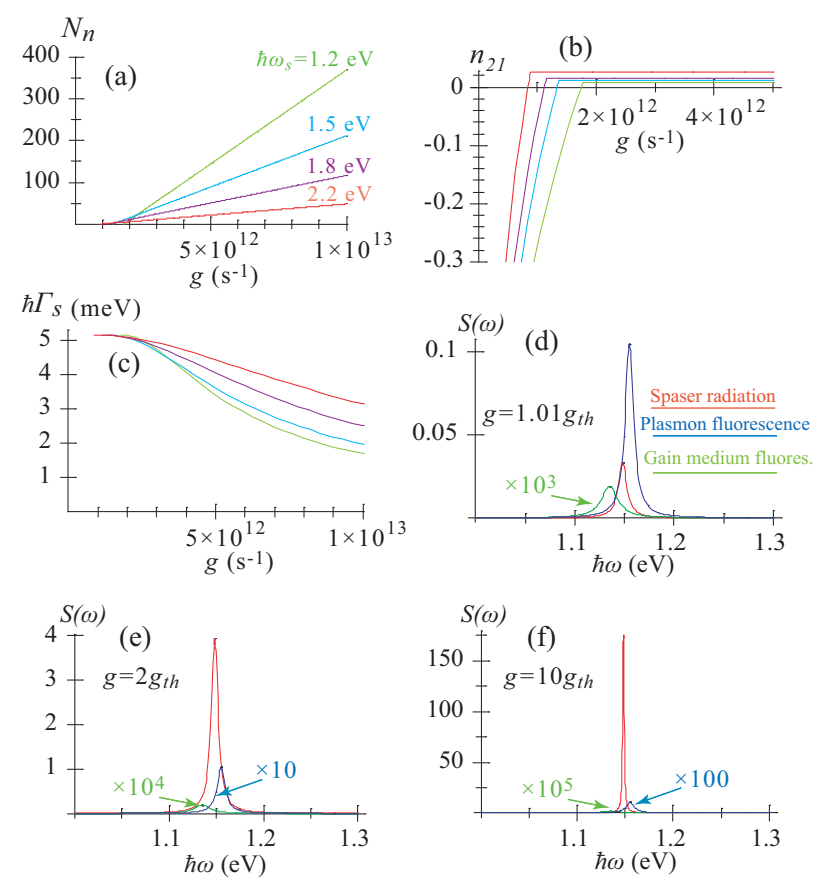

FIG. 2: Spaser SP population and spectral characteristics in the stationary state. The computations are done for a silver nanoshell with the external radius $R_{2}=12 \mathrm{~nm}$; the detuning of the gain medium from the spasing SP mode is $\hbar\left(\omega_{21}-\omega_{n}\right)=-0.02 \mathrm{eV}$. The other parameters are indicated in Sec. II (a) Number $N_{n}$ of plasmons per spasing mode as a function of the excitation rate $g$ (per one chromophore of the gain medium). Computations are done for the dipole eigenmode with the spasing frequencies $\omega_{s}$ as indicated, which were chosen by the corresponding adjustment of the nanoshell aspect ratio. (b) population inversion $n_{12}$ as a function of the pumping rate $g$. The color coding of the lines is the same as in panel (a). (c) The spectral width $\Gamma_{s}$ of the spasing line (expressed as $\hbar \Gamma_{s}$ in meV) as a function of the pumping rate $g$. The color coding of the lines is the same as in panel (a). (d)(f) Spectra of the spaser for the pumping rates $g$ expressed in the units of the threshold rate $g_{t h}$, as indicated in the panels. The curves are color coded and scaled as indicated.

is displayed in Fig. 2 (c) as a function of the pumping rate $g$. At the threshold, $\Gamma_{s}$ is that of the SP line $\gamma_{n}$ but for stronger pumping, as the SPs accumulate in the spasing mode, it decreases $\propto N_{n}^{-1}$, as given by Eq. (8). This decrease of $\Gamma_{s}$ reflects the higher coherence of the spasing state with the increased number of SP quanta and, correspondingly, lower quantum fluctuations. This is similar to the lasers as described by the SchawlowTownes theory ${ }^{43}$.

The developed spasing in a dipole SP mode will show itself in the far field as an anomalously narrow and intense radiation line. The shape and intensity of this line in relation to the lines of the spontaneous fluorescence of the isolated gain medium and its SP-enhanced fluorescence line in the spaser is illustrated in Figs. 2 (d)-(f). Note that for the system under consideration, there is a $20 \mathrm{meV}$ red shift of the gain medium fluorescence with respect to the SP line center. It is chosen so to illustrate the spectral walk-off of the spaser line. For one percent in the excitation rate above the threshold of the spasing [panel (d)], a broad spasing line (red color) appears comparable in intensity to the SP-enhanced spontaneous fluorescence line (blue color). The width of this spasing line is approximately the same as of the fluorescence, but its position is shifted appreciably (spectral walk-off) toward the isolated gain medium line (green color). For the pumping twice more intense [panel (e)], the spaserline radiation dominates, but its width is still close to that of the SP line due to significant quantum fluctuations of the spasing state phase. Only when the pumping rate is an order of magnitude above the threshold, the spaser line strongly narrows [panel (f)], and it also completely dominates the spectrum of the radiation. This is a regime of small quantum fluctuations, which is desired in applications.

These results in the spasing region are different in the most dramatic way from previous phenomenological models, which are based on the consideration of a gain medium that has negative imaginary part of its permittivity plus lossy metal nanosystem, described purely electrodynamically 22.31 . For instance, in a "toy model" 31 , the width of the resonance line tends to zero at the threshold of spasing and then broadens up again. This distinction of the present theory is due the nature of the spasing as a spontaneous symmetry breaking (nonequilibrium phase transition) leading to the establishment of the coherent SP state. This state is influenced by the phase relaxation due to the spontaneous SP emission into the spasing mode leading to the finite spectral width of the spasing line $\Gamma_{s} \propto N_{n}^{-1}$. Therefore the present theory requires a quantum mechanical consideration of the gain medium. Note that below the spasing threshold, the phenomenological theories 22,32 are applicable, describing such important effects as loss compensation in metamaterials $\underline{44}$.

\section{B. Bistable CW Spaser}

Bistables in microelectronics are MOSFET-based devices that have two stable states describing logical 1 and 0 . Their output "logical" level changes when the input exceeds a certain threshold value. Below we show that the spaser can operate as a bistable based on the quantum amplification.

Generally, bistability is a result of nonlinearity in the system. We examine below the bistability in the spaser where the nonlinearity is due to the presence of a saturable absorber. Such an absorber is a chromophore whose absorption overlaps with the spasing line, but which does not directly absorb the radiation that pumps the spaser. Formally, it is described by Eqs. (12), (13) where the pumping rate $g=0$ for the index $p$ corresponding to the saturable absorber. We will assume that this absorber is distributed in the space the same way as the 

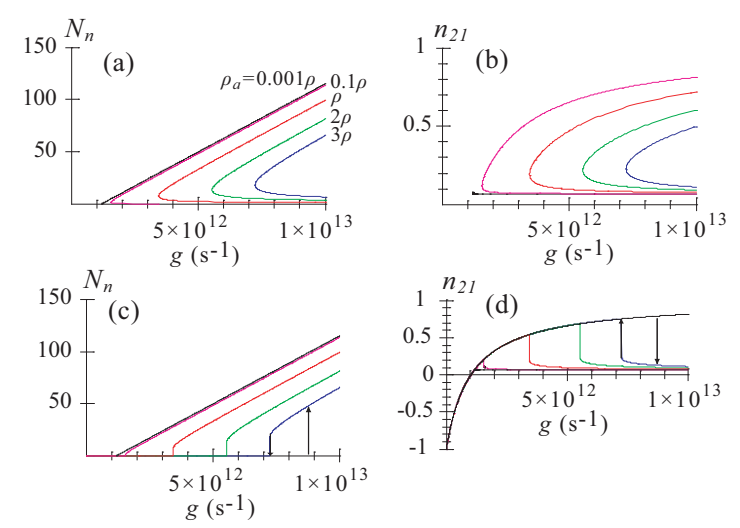

FIG. 3: Bistability in spaser with saturable absorber in a stationary spasing state. (a) Dependence of the SP population number $N_{n}$ in the spasing mode on the pumping rate $g$ for different concentrations $\rho_{a}$ of the saturable absorber. The curves are color coded corresponding to $\rho_{a}$ shown in the units of the concentration $\rho$ of the active medium chromophores. The black line shows the threshold curve (separatrix) between the bistable and uniquely stable solutions. (b) The dependence of the population inversion $n_{21}$ on the pumping rate $g$ for the spasing states. The color coding is the same as in panel (a). Note that the lower branches of the curves in this panel correspond to the upper ones in panel (a) and vice versa. (c) Same as in panel (a) but only the stable branches of the curves are displayed, illustrating the physical behavior of the spaser population $N_{n}$. Note that one of the stable branches coincides with the horizontal axis. The vertical arrows illustrate the hysteretic transitions between the two stable branches for $\rho_{a}=3 \rho$. (d) Same as in panel (c) but for the population inversion $n_{21}$. The black curve starting with $n_{21}=-1$ shows a trivial stable solution corresponding to the absence of spasing $\left(N_{n}=0\right)$.

gain chromophores but with a different density $\rho_{a}$.

Results of a numerical solution of Eqs. (12), (13) for different values of $\rho_{a}$ relatively to the concentration $\rho$ of the gain medium chromophores are shown in Fig. 3 , Panel (a) displays dependence of the SP population number $N_{n}$ in the spasing mode on the pumping rate $g$, and the panel (b) shows the corresponding dependence of the population inversion $n_{21}$ of the gain medium. We note first that there always exists also a trivial, nonspasing solution $N_{n}=0$. For values of $g$ above critical (depending on $\rho_{a}$ ), there are also nontrivial solutions. For $\rho_{a}>10^{-3} \rho$, these nontrivial solutions consist of two branches: high- $N_{n}$ and low- $N_{n}$. Note that the high- $N_{n}$ branch in panel (a) corresponds to the low- $n_{21}$ branch in panel (b), and vice versa.

Thus it appears that there is a tri-stability. However, this impression is incorrect. The lower branches in Fig. 3 (a) [and, correspondingly, the upper branches in panel (b)] describe unstable solutions that are not realizable physically. This can be understood already from the fact that along these branches the SP population decreases with increasing pumping, which is completely unphysical.

It is a bistability, not the tri-stability, which takes place in actuality. This is illustrated in Figs. 3 (c) and (d) obtained by isolating the stable branches of the solutions. As one can see, with an increase of the pumping rate $g$, the solutions appear at critical pumping rates that increase with the saturable absorber concentration $\rho_{a}$. As the critical $g$ for a given $\rho_{a}$ is reached, the nonzero- $N_{n}$ branch appears with a discontinuity. Both the $N_{n}=0$ and $N_{n}>0$ branches are stable and can keep their states indefinitely. The transition between these two stable states can be induced by either adding SP quanta to or removing them from the spasing mode, as illustrated by arrows for $\rho_{a}=3 \rho$. This shows that a spaser with a saturable absorber can serve functions of both a nanoscopic memory cell and a bistable based on quantum amplification. The dynamics of such a device is femtosecond as we show below in Sec. IV

In the bistable case there is no perfect pinning of the population inversion $n_{21}$, as Fig. 3 (d) shows. When the pumping rate $g$ is increased, the system moves along the stable no-spasing $\left(N_{n}=0\right)$ solution denoted by the black line, where the inversion $n_{21}$ significantly overshoots its values for the spasing branches (colored lines). When the transition to spasing occurs, induced, e.g., by an injection of SPs quanta, its is always discontinuous, as the vertical arrows indicate. Along the spasing $\left(N_{n}>0\right)$ branches (colored lines), the inversion counterintuitevely decreases with increased pumping due to the stimulated emission.

It is instructive to compare the behavior of the spaser shown above in Fig. 3 (see also its discussion) with the corresponding behavior of lasers. As we have already mentioned in Sec. I, there are similarities and also substantial differences. A principal source of these differences is due to the fact the spaser SP modes are much stronger localized than the photonic modes in conventional lasers and even the SPP modes in the nanolasers of Refs. 18, 19. The much smaller modal volume $V_{n}$ of the spasing SP modes causes much higher local fields of these SPs [cf. Fig. 1] and, consequently, much stronger feedback in the spaser - cf. Eq. (15). Therefore the spaser operates, including the bistable operation, at many orders of magnitude smaller population of SPs (typically, $\left.N_{n} \sim 100\right)$ per spasing mode as compared to the number of quanta in the resonators of the conventional lasers $\left(\sim 10^{18}-10^{20}\right)$ and even the polaritonic nanolasers. Related to this fact are the very high values of the local fields in the spaser $\sim 10^{6} \sqrt{N_{n}} \sim 10^{7} \mathrm{~V} / \mathrm{cm}$, as is pointed out above in this paragraph. These strong local fields and the strong feedback caused by them are also responsible for the pronounced linear dependence of the SP population $N_{n}$ on the pumping rate [see Fig. 3 (c)] and the pronounced pinning of the population inversion $n_{21}$ of the gain medium [see Fig. 2 (b) and Fig. 3 (d)] in the spasing state. 

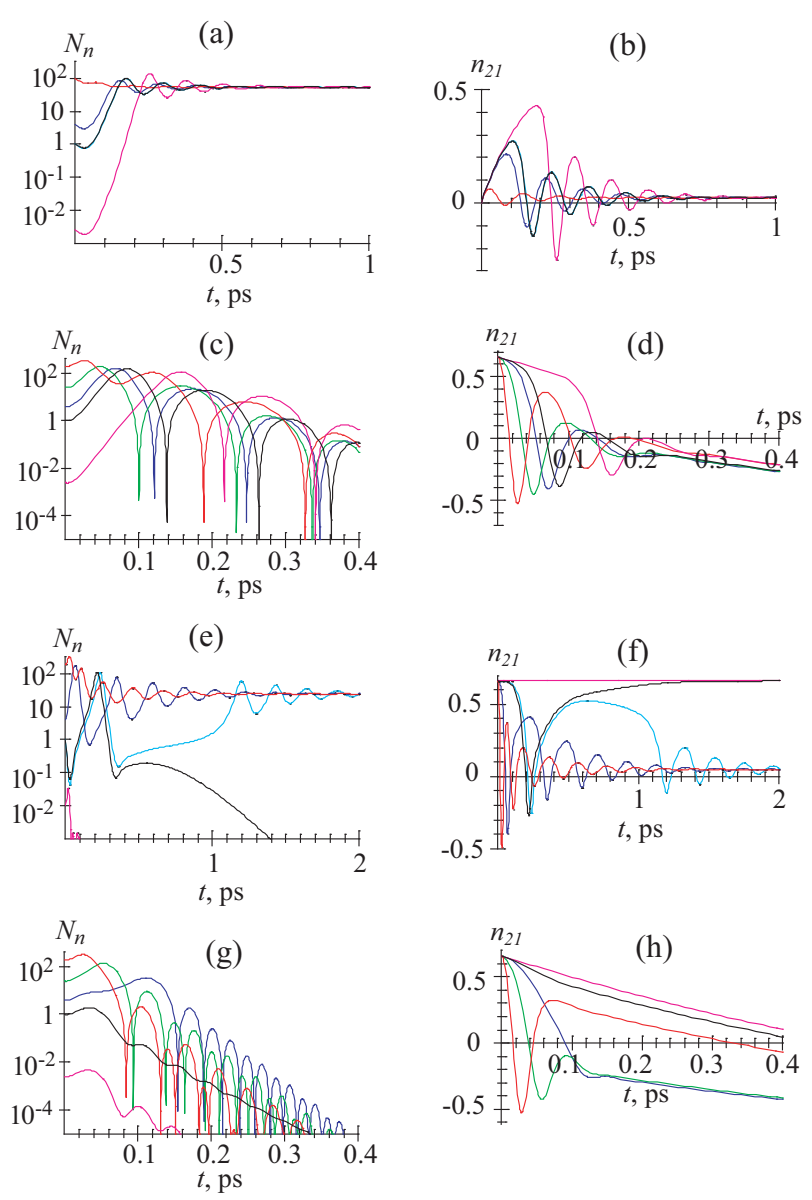

FIG. 4: Ultrafast dynamics of spaser. (a) For monostable spaser (without a saturable absorber), dependence of SP population in the spasing mode $N_{n}$ on time $t$. The spaser is stationary pumped at a rate of $g=5 \times 10^{12} \mathrm{~s}^{-1}$. The color-coded curves correspond to the initial conditions with the different initial SP populations, as shown in the graphs. (b) The same as (a) but for the temporal behavior of the population inversion $n_{21}$. (c) Dynamics of a monostable spaser (no saturable absorber) with the pulse pumping described as the initial inversion $n_{21}=0.65$. Coherent SP population $N_{n}$ is displayed as a function of time $t$. Different initial populations are indicated by color-coded curves. (d) The same as (c) but for the corresponding population inversion $n_{21}$. (e) The same as (a) but for bistable spaser with the saturable absorber in concentration $\rho_{a}=0.66 \rho$. (f) The same as (b) but for the bistable spaser. (g) The same as (e) but for the pulse pumping with the initial inversion $n_{21}=0.65$. (h) The same as (g) but for the corresponding population inversion $n_{21}$.

\section{SPASER AS ULTRAFAST QUANTUM NANOAMPLIFIER}

\section{A. Problem of Setting Spaser as an Amplifier}

Now we consider the central point of this article: setting the spaser as an ultrafast quantum nanoamplifier. As we have already mentioned in the Introduction (Sec. II), the principal and formidable problem is that, in con- trast to the conventional lasers and amplifiers in quantum electronics, the spaser has an inherent feedback that cannot be removed. Thus the spaser will always develop generation and accumulation of the macroscopic number of coherent SPs in the spasing mode. This leads to the the population inversion pinning in the $\mathrm{CW}$ regime at a very low level - cf. Fig. 2 (b). This CW regime corresponds to the net amplification equal zero, which means that the gain exactly compensates the loss, which condition is expressed by Eq. (12). This is a consequence of the nonlinear gain saturation. This holds for any stable CW generator (including any spaser or laser) and precludes using them as amplifiers.

We propose two regimes for setting the spaser as a quantum nanoamplifier. The first is a transient regime based on the fact that the establishment of the CW regime and the consequent inversion pinning and total gain vanishing requires some time that is determined mainly by the rate of the quantum feedback, but depends also on the relaxation rates of the SPs and the gain medium. After the population inversion is created by the onset of pumping and before the spasing spontaneously develops, as we show below in this Section, there is a time interval of approximately $250 \mathrm{fs}$, during which the spaser provides usable (and as predicted, quite high) amplification.

The second way to set the spaser as a quantum nanoamplifier is a bistable regime that is achieved by introducing a saturable absorber, which prevents the spontaneous spasing. Then injection of a certain abovethreshold amount of SP quanta will saturate the absorber and initiate the spasing. Such a bistable quantum amplifier will be considered in the subsequent Subsection. The bistability is the most promising regime which allows not only the functioning of the spaser as a ultrafast threshold (logical) amplifier, but also a quasi-CW bistability where the spaser works as a memory element [cf. Sec. IIIB] with an ultrafast switching time.

The temporal behavior of the spaser has been found by direct numerical solution of Eqs. (4)-(16). This solution is facilitated by the fact that in the model under consideration all the chromophores experience the same local field inside the nanoshell, and there are only two types of such chromophores: belonging to the gain medium and the saturable absorber, if it is present.

\section{B. Monostable Spaser as a Nanoamplifier in Transient Regime}

In this Subsection we consider a monostable spaser in a transiebt regime. This implies that no saturable absorber is present. We will consider two pumping regimes: stationary and pulse.

Starting with the stationary regime, we assume that the pumping at a rate (per one chromophore) of $g=$ $5 \times 10^{12} \mathrm{~s}^{-1}$ starts at a moment of time $t=0$ and stays constant after that. Immediately at $t=0$, a certain num- 
ber of SPs are injected into the spaser. We are interested in its temporal dynamics from this moment on.

The dynamical behavior of the spaser under this pumping regime is illustrated in Figs. 4 (a), (b). As we see, the spaser, which starts from an arbitrary initial population $N_{n}$, rather rapidly, within a few hundred femtoseconds approaches the same stationary ("logical") level. At this level, an SP population of $N_{n}=67$ is established, while the inversion is pinned at a low level of $n_{21}=0.02$. On the way to this stationary state, the spaser experiences relaxation oscillations in both the SP numbers and inversion, which have a trend to oscillate out of phase [compare panels (a) and (b)]. This temporal dynamics of the spaser is quite complicated and highly nonlinear (unharmonic). It is controlled not by a single relaxation time but by a set of the relaxation rates. Clearly, among these are the energy transfer rate from the gain medium to the SPs and the relaxation rates of the SPs and the chromophores.

In this mode, the main effect of the initial injection of the SPs (described theoretically as different initial values of $N_{n}$ ) is in the interval of time it is required for the spaser to reach the final $(\mathrm{CW})$ state. For very small $N_{n}$, which in practice can be supplied by the noise of the spontaneous SP emission into the mode, this time is approximately 250 fs (cf.: the corresponding SP relaxation time is less then $50 \mathrm{fs}$ ). In contrast, for the initial values of $N_{n}=1-5$, this time shortens to $150 \mathrm{fs}$. Whether this is a practically usable difference remains to be seen at more advanced stages of development.

Now consider the second regime: pulse pumping. The gain-medium population of the spaser is inverted at $t=0$ to saturation with a short (much shorter than $100 \mathrm{fs}$ ) pump pulse. Simultaneously, at $t=0$, some number of plasmons are injected (say, by an external nanoplasmonic circuitry). In response, the spaser should produce an amplified pulse of the SP excitation. Such a function of the spaser is illustrated in Figs. 4 (c) and (d).

As we see from panel (c), independently from the initial number of SPs, the spaser always generates a series of SP pulses, of which only the first pulse is large (at or above the logical level of $N_{n} \sim 100$ ). (An exception is a case of little practical importance when the initial $N_{n}=120$ exceeds this logical level, when two large pulses are produced.) The underlying mechanism of such a response is the rapid depletion of the inversion seen in panel (d), where energy is dissipated in the metal of the spaser. The characteristic duration of the SP pulse $\sim 100 \mathrm{fs}$ is defined by this depletion, controlled by the energy transfer and $\mathrm{SP}$ relaxation rates. This time is much shorter than the spontaneous decay time of the gain medium. This acceleration is due to the stimulated emission of the SPs into the spasing mode (which can be called a "stimulated Purcell effect"). There is also a pronounced trend: the lower is initial SP population $N_{n}$, the later the spaser produces the amplified pulse. In a sense, this spaser functions as a pulse-amplitude to time-delay converter.

\section{Bistable Spaser (with Saturable Absorber) as an Ultrafast Nanoamplifier}

Now let us consider the most important point of this article: a bistable spaser as a quantum threshold ("logical") nanoamplifier. Such a spaser contains a saturable absorber mixed with the gain medium with parameters indicated at the end of Sec. IIA In particular, the concentration of the saturable absorber $\rho_{a}=0.66 \rho$. This case of a bistable spaser amplifier is of a particular interest because in this regime the spaser comes as close as possible in its functioning to the semiconductor-based (mostly, MOSFET-based) digital nanoamplifiers. As in the previous Subsection, we will consider two cases: the stationary and short-pulse pumping.

We again start with the case of the stationary pumping at a rate of $g=5 \times 10^{12} \mathrm{~s}^{-1}$. We show in Figs. 4 (e), (f) the dynamics of such a spaser. For a small initial population $N_{n}=5 \times 10^{-3}$ simulating the spontaneous noise, the spaser is rapidly (faster than in $50 \mathrm{fs}$ ) relaxing to the zero population [panel (e)], while its gain-medium population is equally rapidly approaching a high level [panel (f)] $n_{21}=0.65$ that is defined by the competition of the pumping and the enhanced decay into the SP mode (the purple curves). This level is so high because the spasing SP mode population vanishes and the stimulated emission is absent. After reaching this stable state (which one can call, say, "logical zero"), the spaser stays in it indefinitely long despite the continuing pumping.

In contrast, for initial values $N_{n}$ of the SP population large enough [for instance, for $N_{n}=5$, as shown by the blue curves in Figs. 4 (e) and (f)], the spaser tends to the "logical one" state where the stationary SP population reaches the value of $N_{n} \approx 60$. Due to the relaxation oscillations, it actually exceeds this level within a short time of $\lesssim 100$ fs after the seeding with the initial SPs. As the SP population $N_{n}$ reaches its stationary (CW) level, the gain medium inversion $n_{21}$ is pinned down at a low level of a few percent, as typical for the $\mathrm{CW}$ regime of the spaser. This "logical one" state salso persists indefinitely, as long as the inversion is supported by the pumping.

There is a critical curve (separatrix) that divide the two stable dynamics types (leading to the logical levels of zero and one). For the present set of parameters this separatrix starts with the initial population of $N_{n} \approx 1$. For a value of the initial $N_{n}$ slightly below 1 , the SP population $N_{n}$ experiences a slow (hundreds fs in time) relaxation oscillation but eventually relaxes to zero [Fig. 4](e), black curve], while the corresponding chromophore population inversion $n_{21}$ relaxes to the high value $n_{21}=0.65$ [panel (f), black curve]. In contrast, for a value of $N_{n}$ slightly higher than 1 [light blue curves in panels (e) and (f)], the dynamics is initially close to the separaratrix but eventually the initial slow dynamics tends to the high SP population and low chromophore inversion through a series of the relaxation oscillations. The dynamics close to the separatrix is characterized by a wide range of oscillation times due to its highly nonlinear character. The 
initial dynamics is slowest (the "decision stage" of the spaser bistable that lasts $\gtrsim 1 \mathrm{ps)}$. The "decision time" is diverging infinitesimally close to the separatrix, as is characteristic of any threshold (logical) amplifier.

The gain (amplification coefficient) of the spaser as a threshold (logical or bistable) amplifier is the ratio of the high CW level to the threshold level of the SP population $N_{n}$. For this specific spaser with the chosen set parameters, this gain is $\approx 60$, which is more than sufficient for the digital information processing. Thus this spaser can make a high-gain, $\sim 10 \mathrm{THz}$-bandwidth logical amplifier or dynamical memory cell with excellent prospects of applications.

The last but probably the most important regime to consider is that of the pulse pumping in the bistable spaser. In this case, the population inversion $\left(n_{21}=\right.$ $0.65)$ is created by a short pulse at $t=0$ and simultaneously initial SP population $N_{n}$ is created. Both are emulated as the initial conditions in Eqs. (44)-(6). The corresponding results are displayed in Figs. 4 (g) and (h).

When the initial SP population exceeds the critical one of $N_{n}=1$ (the blue, green, and red curves), the spaser responds with generating a short (duration less than $100 \mathrm{fs}$ ) pulse of the SP population (and the corresponding local fields) within a time $\lesssim 100$ fs [panel (g)]. Simultaneously, the inversion is rapidly (within $\sim 100 \mathrm{fs}$ ) exhausted [panel (h)].

In contrast, when the initial SP population $N_{n}$ is less than the critical one (i.e., $N_{n}<1$ in this specific case), the spaser rapidly (within a time $\lesssim 100$ fs) relaxes as $N_{n} \rightarrow 0$ through a series of realaxation oscillations - see the black and magenta curves in Fig. $4(\mathrm{~g})$. The corresponding inversion decays in this case almost exponentially with a characteristic time $\sim 1$ ps determined by the enhanced energy transfer to the SP mode in the metal see the corresponding curves in panel (h). Note that the SP population decays faster due to the threshold nature of spasing.

Gold as the spaser nanoplasmonic-core metal, which is used in experiments 16 , has the relaxation rate an order magnitude higher than silver. Therefore it is plausible that a gold-core spaser as an amplifier will have a bandwidth $\sim 100 \mathrm{THz}$. We will consider such a system elsewhere. Taking into account that the local fields produced by the spaser are concentrated within a few tens of nanometers in its vicinity, these are parameters that bear promise of many applications in the fundamental science and coming digital and analog femtosecond technologies.

\section{DISCUSSION AND CONCLUSION}

We have demonstrated principal possibilities of the spaser functioning as a ultrafast nanoamplifier of the local optical fields. In doing so we have had to overcome a principal and formidable problem. Any spaser has the inherent feedback. Consequently, the spaser as proposed initially $\underline{\underline{4}}$ and observed experimentally $\underline{16}$ rapidly, on the femtosecond time scale, will generate the coherent population of the SPs and approach the CW regime. In this regime, the net gain (the saturated amplification minus loss) is exactly zero (the same as for the lasers). Thus CW spasers (or CW lasers, for that matter) principally cannot be used as amplifiers. In the conventional optical amplifiers, in contrast to lasers, the feedback is deliberately removed and any parasitic feedback (scattering, etc.) is carefully minimized to prevent the spontaneous generation. Such a regime is not possible for the spaser as it is presently known. In this article, we have demonstrated two ways to set a spaser as a nanoamplifier: (i) the conventional monostable spaser can amplify in the transient ultrafast (femtosecond) regime before the CW generation is established; (ii) the spaser with the saturable absorber can become bistable and function as threshold (logical) amplifier with ultrafast (femtosecond) switching times.

We have developed quantum theory of the spaser based on density matrix (optical Bloch) equations. The spaser described is a nanoparticle that consists of the metal core and the gain (active) medium. The metal core plays the role of the resonator ("cavity") whose states are the localized SPs, which we have quantized. The gain medium is described completely quantum mechanically taking into account the excitation, decay, and dephasing processes. The main approximation that we have employed is that the SP population number $N_{n}$ is treated as a classical variable. This is the same semiclassical approximation that is most often used in the physics of lasers. Given the fact that the typical number of the SP quanta per spasing mode is still large enough $\left(N_{n} \sim 100\right)$, this approximation appears reasonable and reliable.

Pursuing the goal of the fastest possible operation and the nanometric size (on the same order as that of microelectronic MOSFETs), we have deliberately considered spasers whose size is much less than the radiation wavelength and whose metal thickness is less than the skin depth. This has allowed us to use the quasistatic SP eigenmodes as full counterparts of the electrostatic fields in the transistors. Note that such nanoscopic a spaser has recently been demonstrated experimentally 16 .

Though the main emphasis of this article is on the ultrafast dynamics of the spaser as a nanoamplifier of the local plasmonic fields, the CW regime is described first as the basis of the understanding the ultrafast dynamics. The corresponding results are illustrated in Fig. 2, The "spasing curve" (the dependence of the SP population $N_{n}$ on the pumping rate) shows a pronounced threshold and a linear dependence of the coherent SP population $N_{n}$ on the pumping rate $g$ [Fig. 2(a)]. This linear dependence $N_{n}(g)$ is due to the extremely strong feedback in the spaser caused by the very strong SP mode localization. The same effect is responsible for clipping the population inversion in the spasing state to very low levels, as illustrated in Fig. 2(b). With the increased pumping, a higher SP population leads to lower quantum fluctuations and decreased spectral width of the spasing line, 
as shown in Fig. 2 (c). Gradually, a very narrow and intense spasing line appears in the emission spetrum of the spaser - see Figs. 2 (d)-(f). This narrow line is indicative of the intense optical fields that are excited in the vicinity of a spaser - cf. Fig. 1(a) where the field must be multiplied by a factor of $\sqrt{N_{n}}$. Qualitatively, the linear spasing curve and the narrowing of the spasing line with the pumping are in a full agreement with the recent experiment ${ }^{16}$.

Adding a saturable absorber to the spaser shell, we have shown that it can work as a bistable based on quantum amplification. The corresponding results are illustrated in Fig. 3 for a CW operation mode.

The main results of this article concern with ultrafast processes of amplification in the spaser. These are illustrated in Fig. [4. We have shown that a monostable spaser (without a saturable absorber) performs amplification of the injected SPs in a transient regime. However, this process only manifests itself in the shorter times to establish the high SP population - see Figs. 4 (a)-(d) and the corresponding discussion. In this sense, such a spaser performs the function of the amplitude to time converter but not a true nanoamplifier.

The most significant results are those related to the ultrafast amplification in the bistable spaser containing a saturable absorber within its gain shell. This amplification has many similarities with that in semiconductor threshold (logical) nanoamplifiers based on the MOSFETs. Such a spaser with a stationary pumping behaves as an ultrafast nanoscale bistable - see results in Figs. 4 (e) and (f). For the initial conditions not too close to the threshold, it amplifies and switches with a characteristic time of $\sim 100 \mathrm{fs}$. The bistable spaser with the pulse pumping is a high-gain ultrafast pulse nanoamplifier see Figs. $4(\mathrm{~g})$ and $(\mathrm{h})$ and the corresponding discussion in the previous Section. Its typical response time and pulse length are $\lesssim 100 \mathrm{fs}$, which correspond to the 10 $\mathrm{THz}$ bandwidth.

For the gold-based spaser, in contrast to the present silver-based one, the typical switching time should be much shorter, presumably $\sim 10$ fs corresponding to $\sim 100$ $\mathrm{THz}$ bandwidth. However, the gold-based spasers will work at a lower SP population due to the higher loss in the metal. Correspondingly, they will possess lower signal/noise ratio and wider spectral lines. We will consider such spasers elsewhere.

Concluding, the spaser is the size of the MOSFET 13 $(\sim 10 \mathrm{~nm})$ and can perform the same function of signal amplification on the nanoscale. Having a high enough gain, the spaser can be an active-element foundation of highly integrated nanoplasmonic devices, including ultrafast processors. Based on metals, it is inherently much faster, by a factor of $\sim 1000$ than silicon devices, as we have shown above. For the same reason, it also highly robust environmentally: it can work at high temperatures, in the presence of microwave and ionizing radiations, etc. This article has presented quantum theory of the spaser as a nanoamplifier, which is critically needed for understanding and utilization of the spaser.

This work was supported by grants from the Chemical Sciences, Biosciences and Geosciences Division of the Office of Basic Energy Sciences, Office of Science, U.S. Department of Energy, a grant CHE-0507147 from NSF, and a grant from the US-Israel BSF. Work at Garching was supported under contract from Ludwig Maximilian University of Munich (Germany) in the framework of the Munich Advanced Photonics Center (MAP). I appreciate useful discussions with S. Tikhodeev regarding bistability in lasers.
* Electronic address: mstockman@gsu.edu URL: http://www .phy-astr.gsu.edu/stockman

${ }^{1}$ L. Novotny and B. Hecht, Principles of Nano-Optics (Cambridge University Press, Cambridge, New York, 2006).

2 M. I. Stockman, M. F. Kling, U. Kleineberg, and F. Krausz, Nature Photonics 1, 539 (2007).

3 M. I. Stockman, New J. Phys. 10, 025031 (2008).

${ }^{4}$ D. J. Bergman and M. I. Stockman, Phys. Rev. Lett. 90, 027402 (2003).

${ }^{5}$ H. A. Atwater, Sci. Am. 296, 56 (2007).

6 J. N. Anker, W. P. Hall, O. Lyandres, N. C. Shah, J. Zhao, and R. P. V. Duyne, Nature Materials 7, 442 (2008).

7 A. Israel, M. Mrejen, Y. Lovsky, M. Polhan, S. Maier, and A. Lewis, Laser Focus World 43, 99 (2007).

8 L. Tang, S. E. Kocabas, S. Latif, A. K. Okyay, D. S. LyGagnon, K. C. Saraswat, and D. A. B. Miller, Nat. Phot. 2, 226 (2008).

9 W. A. Challener, C. Peng, A. V. Itagi, D. Karns, W. Peng, Y. Peng, X. Yang, X. Zhu, N. J. Gokemeijer, Y. T. Hsia, et al., Nat. Phot. 3, 220 (2009).
10 S. Kim, J. H. Jin, Y. J. Kim, I. Y. Park, Y. Kim, and S. W. Kim, Nature 453, 757 (2008).

11 N. Nagatani, R. Tanaka, T. Yuhi, T. Endo, K. Kerman, and Y. T. and. E Tamiya, Science and Technology of Advanced Materials 7, 270 (2006).

12 L. R. Hirsch, R. J. Stafford, J. A. Bankson, S. R. Sershen, B. Rivera, R. E. Price, J. D. Hazle, N. J. Halas, and J. L. West, Proc. Natl. Acad. Sci. USA 100, 13549 (2003).

13 D. Kahng, United States Patent 3,102,230 (1963).

14 Y. Tsividis, Operation and Modeling of the MOS Transistor (McGraw-Hill, New York, 1999).

15 M. I. Stockman, Nat. Phot. 2, 327 (2008).

16 M. A. Noginov, G. Zhu, A. M. Belgrave, R. Bakker, V. M. Shalaev, E. E. Narimanov, S. Stout, E. Herz, T. Suteewong, and U. Wiesner, Nature 460, 1110 (2009).

17 M. T. Hill, Y.-S. Oei, B. Smalbrugge, Y. Zhu, T. d. Vries, P. J. v. Veldhoven, F. W. M. v. Otten, T. J. Eijkemans, J. a. P. Turkiewicz, H. d. Waardt, et al., Nat. Phot. 1, 589 (2007).

18 M. T. Hill, M. Marell, E. S. P. Leong, B. Smalbrugge, Y. Zhu, M. Sun, P. J. van Veldhoven, E. J. Geluk, 
F. Karouta, Y.-S. Oei, et al., Opt. Express 17, 11107 (2009).

19 R. F. Oulton, V. J. Sorger, T. Zentgraf, R.-M. Ma, C. Gladden, L. Dai, G. Bartal, and X. Zhang, Nature 461, 629 (2009).

20 M. I. Stockman, S. V. Faleev, and D. J. Bergman, Phys. Rev. Lett. 87, 167401 (2001).

21 I. A. Larkin and M. I. Stockman, Nano Lett. 5, 339 (2005).

22 J. A. Gordon and R. W. Ziolkowski, Opt. Expr. 15, 2622 (2007).

23 D. J. Bergman and D. Stroud, in Solid State Physics, edited by H. Ehrenreich and D. Turnbull (Academic Press, Boston, 1992), vol. 46, pp. 148-270.

24 D. J. Bergman and D. Stroud, Phys. Rev. B 22, 3527 (1980).

25 A. S. Kirakosyan, M. I. Stockman, and T. V. Shahbazyan, ArXiv:0908.0647 (2009).

${ }^{26}$ E. Plum, V. A. Fedotov, P. Kuo, D. P. Tsai, and N. I. Zheludev, Opt. Expr. 17, 8548 (2009).

27 J. Seidel, S. Grafstroem, and L. Eng, Phys. Rev. Lett. 94, 177401 (2005).

28 M. A. Noginov, G. Zhu, M. Mayy, B. A. Ritzo, N. Noginova, and V. A. Podolskiy, Phys. Rev. Lett. 101, 226806 (2008).

29 K. Li, X. Li, M. I. Stockman, and D. J. Bergman, Phys. Rev. B 71, 115409 (2005).

30 Z. G. Dong, H. Liu, T. Li, Z. H. Zhu, S. M. Wang, J. X. Cao, S. N. Zhu, and X. Zhang, Opt. Expr. 16, 20974 (2008).

31 M. Wegener, J. L. Garcia-Pomar, C. M. Soukoulis, N. Meinzer, M. Ruther, and S. Linden, Opt. Expr. 16, 19785 (2008).
32 A. Fang, T. Koschny, M. Wegener, and C. M. Soukoulis, Phys. Rev. B (Rapid Communications) 79, 241104(R) (2009).

33 S. W. Chang, C. Y. A. Ni, and S. L. Chuang, Opt. Expr. 16, 10580 (2008).

34 N. I. Zheludev, S. L. Prosvirnin, N. Papasimakis, and V. A. Fedotov, Nat. Phot. 2, 351 (2008).

35 I. E. Protsenko, A. V. Uskov, O. A. Zaimidoroga, V. N. Samoilov, and E. P. O'Reilly, Phys Rev A 71, 063812 (2005).

36 M. Ambati, S. H. Nam, E. Ulin-Avila, D. A. Genov, G. Bartal, and X. Zhang, Nano Lett. 8, 3998 (2008).

37 Z. K. Zhou, X. R. Su, X. N. Peng, and L. Zhou, Opt. Expr. 16, 18028 (2008).

38 M. A. Noginov, V. A. Podolskiy, G. Zhu, M. Mayy, M. Bahoura, J. A. Adegoke, B. A. Ritzo, and K. Reynolds, Opt. Expr. 16, 1385 (2008).

${ }^{39}$ G. Adamo, K. F. MacDonald, Y. H. Fu, C. M. Wang, D. P. Tsai, F. J. G. de Abajo, and N. I. Zheludev, Phys. Rev. Lett. 103, 113901 (2009).

40 M. A. Noginov, G. Zhu, M. Bahoura, J. Adegoke, C. Small, B. A. Ritzo, V. P. Drachev, and V. M. Shalaev, Appl. Phys. B 86, 455 (2007).

41 M. A. Noginov, J. Nanophotonics 2, 021855 (2008).

42 P. B. Johnson and R. W. Christy, Phys. Rev. B 6, 4370 (1972).

43 A. L. Schawlow and C. H. Townes, Phys. Rev. 112, 1940 (1958).

44 V. M. Shalaev, Nature Photonics 1, 41 (2007).

45 Note that we have corrected a misprint in Ref. 4 replacing the coefficient $2 \pi$ by $4 \pi$. 